\title{
SCIENTIFIC REPORTS

\section{OPEN Evaluation of the postoperative stability of a counter-clockwise rotation technique for skeletal class II patients by using a novel three- dimensional position-posture method}

\author{
Zhuqing Wan ${ }^{1}$, Steve Guofang Shen ${ }^{1}$, Haijun Gui ${ }^{1}$, Peng Zhang ${ }^{2}$ \& Shunyao Shen ${ }^{1}$
}

The aim of this study is to evaluate the postsurgical stability of skeletal class II patients after performing a counter-clockwise rotational (CCWR) procedure for the maxilla-mandibular complex (MMC) by using a novel Three-dimensional (3D) Position-Posture(P-P) measuring method. Twenty-five patients ( 5 males and 20 females) were included in this study. The postoperative CT scans of the skull were taken before surgery(T0), 3-7 days (T1), 3 months (T2), and 6 months (T3) after surgery. Specific anatomic landmarks were chosen to determine the position of the segments, while three equally perpendicular planes were created to describe their posture. The results show that the linear relapse of maxillary landmarks during the follow-up were acceptable $(\leq 0.5 \mathrm{~mm})$. The relapse of maxillary pitch plane at 6-months follow-up is $1.52^{\circ}$, which is acceptable. There was a significant pitch plane relapse of the mandibular-body segment with an average of $1.86^{\circ}$ between $\mathrm{T} 1$ and $\mathrm{T} 2$ models, $3.28^{\circ}$ between $\mathrm{T} 1$ and $\mathrm{T} 3$ models. There was no significant difference between roll and yaw planes during the follow-up. We therefore conclude that the P-P method could be used to accurately analyze the postsurgical stability of skeletal class II orthognathic surgery cases. For CCWR procedures, it was also shown that the there is a tendency for recurrence most specially on the body of the mandible.

Skeletal class II malocclusion is a common dentofacial deformity, which is characterized by the retrusive position of the mandible in relation to the maxilla ${ }^{1}$. Orthognathic surgery with counterclockwise rotation (CCWR) of the maxillo-mandibular complex (MMC) can be performed to achieve a more optimal function and esthetically acceptable outcome $\mathrm{e}^{2-4}$.

However, postsurgical stability after CCWR remains a challenge. Several studies have reported the poor postsurgical stability after CCWR. A coexisting TMJ pathology can jeopardize the stability of the MMC after performing the CCWR. Because that the increased joint loading can cause TMJ-associated symptoms, condylar resorption, malocclusion, and relapse ${ }^{5-11}$. Wolford ${ }^{12}$ et al. reported that there is an increased posterior facial height with subsequent stretching of the pterygoid, masseteric, and suprahyoid muscles that could place additional load to the TMJs, with potential adverse results.

Recently, computer-aided design and computer-aided manufacturing technologies have been developed to improve the repeatability, precision and accuracy of orthognathic surgery, giving the potential to realize ideal

${ }^{1}$ Department of Oral and Cranio-maxillofacial Surgery, Shanghai Ninth People's Hospital, College of Stomatology, Shanghai Jiao Tong University School of Medicine Shanghai Key Laboratory of Stomatology \& Shanghai Research Institute of Stomatology No. 639 Zhizaoju Road, Shanghai, 200011, China. ${ }^{2} 2$ nd Dental Center, Shanghai Ninth People's Hospital, Shanghai Jiao Tong University School of Medicine Shanghai Key Laboratory of Stomatology No. 639 Zhizaoju Road, Shanghai, 200011, China. Zhuqing Wan and Steve Guofang Shen contributed equally. Correspondence and requests for materials should be addressed to H.G. (email: yuanqing860409@163.com) or P.Z. (email: zp_kitty@163.com) 


\begin{tabular}{|c|c|c|c|c|c|c|c|}
\hline & T0 & T1 & T1-T0 & T2 & T2-T1 & T3 & T3-T1 \\
\hline & Mean (SD) & Mean (SD) & p value & Mean (SD) & p value & Mean (SD) & p value \\
\hline A-CP $(\mathrm{mm})$ & $1.46(2.91)$ & $-1.65(3.26)$ & $0.000^{*}$ & $-1.28(3.12)$ & 0.321 & $-1.17(2.99)$ & 0.308 \\
\hline A-HP (mm) & $62.87(3.98)$ & $59.44(4.86)$ & $0.000 *$ & $59.13(4.72)$ & 0.866 & $59.33(4.70)$ & 1.000 \\
\hline A-SP (mm) & $0.30(1.02)$ & $0.19(1.23)$ & 1.000 & $0.14(1.25)$ & 1.000 & $-0.03(1.25)$ & 0.071 \\
\hline SPC-CP (mm) & $3.63(2.53)$ & $2.34(2.29)$ & $0.024^{*}$ & $2.36(2.29)$ & 1.000 & $2.13(1.97)$ & 1.000 \\
\hline SPC-HP (mm) & $76.45(3.82)$ & $72.35(4.53)$ & $0.000^{*}$ & $72.51(4.51)$ & 1.000 & $72.32(4.18)$ & 1.000 \\
\hline SPC-SP (mm) & $1.00(0.91)$ & $0.70(0.98)$ & 1.000 & $0.58(0.93)$ & 1.000 & $0.54(0.83)$ & 0.578 \\
\hline SP6L-CP (mm) & $-21.71(3.31)$ & $-23.52(3.66)$ & $0.022 *$ & $-23.17(3.84)$ & 0.600 & $-23.16(3.49)$ & 1.000 \\
\hline SP6L-HP (mm) & $70.11(4.90)$ & $68.10(4.55)$ & $0.002 *$ & $67.94(4.86)$ & 1.000 & $67.86(4.84)$ & 0.785 \\
\hline SP6L-SP (mm) & $29.03(2.01)$ & $28.85(2.14)$ & 1.000 & $28.67(2.12)$ & 0.340 & $28.70(2.21)$ & 1.000 \\
\hline SP6R-CP (mm) & $-20.28(4.43)$ & $-22.51(3.85)$ & $0.001 *$ & $-22.08(3.86)$ & 0.184 & $-22.06(3.78)$ & 0.524 \\
\hline SP6R-HP (mm) & $70.58(4.57)$ & $68.56(4.72)$ & $0.000^{*}$ & $68.21(4.86)$ & 0.118 & $68.27(4.80)$ & 0.462 \\
\hline SP6R-SP (mm) & $-28.74(1.54)$ & $-28.56(1.19)$ & 1.000 & $-28.60(1.28)$ & 1.000 & $-28.55(0.98)$ & 1.000 \\
\hline$\angle \mathrm{PP}-\mathrm{CP}\left({ }^{\circ}\right)$ & $9.88(6.31)$ & $16.36(4.94)$ & $0.000^{*}$ & $15.93(5.19)$ & 1.000 & $14.84(5.41)$ & 0.085 \\
\hline$\angle \mathrm{RP}-\mathrm{HP}\left({ }^{\circ}\right)$ & $0.58(1.95)$ & $0.60(1.86)$ & 1.000 & $0.39(1.91)$ & 0.071 & $0.37(1.83)$ & 0.668 \\
\hline$\angle \mathrm{YP}-\mathrm{CP}\left({ }^{\circ}\right)$ & $0.82(1.97)$ & $0.35(2.19)$ & 1.000 & $0.27(2.46)$ & 1.000 & $0.32(2.25)$ & 1.000 \\
\hline$\angle \mathrm{SNA}\left({ }^{\circ}\right)$ & $84.23(4.13)$ & $81.11(4.53)$ & $0.000 *$ & $81.37(4.52)$ & 1.000 & $81.43(4.57)$ & 0.487 \\
\hline B-CP' (mm) & $71.03(9.79)$ & $73.84(8.45)$ & $0.005^{*}$ & $73.49(9.35)$ & 1.000 & $71.84(10.25)$ & $0.012 *$ \\
\hline B-HP' (mm) & $57.03(9.05)$ & $60.01(9.69)$ & $0.024^{*}$ & $60.27(10.30)$ & 1.000 & $60.69(10.45)$ & 1.000 \\
\hline B-SP' (mm) & $54.57(3.21)$ & $54.53(2.81)$ & 1.000 & $54.01(3.01)$ & 0.161 & $53.77(3.06)$ & $0.034 *$ \\
\hline IPC-CP' (mm) & $75.76(8.45)$ & $77.32(7.54)$ & 0.221 & $77.16(8.63)$ & 1.000 & $75.53(9.39)$ & $0.047^{*}$ \\
\hline IPC-HP' (mm) & $50.05(10.58)$ & $53.22(11.29)$ & $0.004^{*}$ & $53.42(11.84)$ & 1.000 & $54.20(11.73)$ & 0.329 \\
\hline IPC-SP' (mm) & $54.47(3.60)$ & $54.44(3.16)$ & 1.000 & $53.76(3.36)$ & $0.025 *$ & $53.63(3.30)$ & $0.035 *$ \\
\hline IP6L-CP' (mm) & $54.27(5.76)$ & $56.97(3.69)$ & $0.039 *$ & $56.64(4.85)$ & 1.000 & $55.61(5.34)$ & 0.308 \\
\hline IP6L-HP' (mm) & $45.49(7.03)$ & $48.42(7.47)$ & $0.003 *$ & $48.68(7.91)$ & 1.000 & $48.91(8.08)$ & 1.000 \\
\hline IP6L-SP' (mm) & $80.70(3.08)$ & $81.19(2.67)$ & 1.000 & $80.58(3.00)$ & 0.055 & $80.32(2.79)$ & $0.004 *$ \\
\hline IP6R-CP'(mm) & $52.09(11.67)$ & $54.31(11.35)$ & $0.026^{*}$ & $53.77(12.49)$ & 0.984 & $52.53(12.64)$ & $0.034^{*}$ \\
\hline IP6R-HP' (mm) & $45.77(6.98)$ & $49.07(8.00)$ & $0.002 *$ & $49.00(8.06)$ & 1.000 & $49.47(8.14)$ & 1.000 \\
\hline IP6R-SP' (mm) & $26.97(4.74)$ & $27.29(4.36)$ & 1.000 & $26.67(4.49)$ & 0.058 & $26.44(4.49)$ & $0.021 *$ \\
\hline$\angle \mathrm{PP}^{\prime}-\mathrm{CP}^{\prime}\left({ }^{\circ}\right)$ & $31.16(12.16)$ & $26.43(12.16)$ & $0.000 *$ & $28.29(11.65)$ & $0.013 *$ & $29.71(12.31)$ & $0.000 *$ \\
\hline$\angle \mathrm{RP}^{\prime}-\mathrm{HP}^{\prime}\left({ }^{\circ}\right)$ & $1.60(1.34)$ & $1.57(1.35)$ & 1.000 & $1.49(1.15)$ & 1.000 & $1.78(0.95)$ & 1.000 \\
\hline$\angle \mathrm{YP}^{\prime}-\mathrm{CP}^{\prime}\left(^{\circ}\right)$ & $1.91(1.24)$ & $1.50(0.66)$ & 1.000 & $1.76(1.15)$ & 1.000 & $1.66(1.28)$ & 1.000 \\
\hline$\angle \mathrm{RsPL}-\mathrm{RsPR}\left({ }^{\circ}\right)$ & $25.55(10.66)$ & $25.21(10.42)$ & 1.000 & $24.90(10.56)$ & 1.000 & $24.26(10.48)$ & 0.069 \\
\hline
\end{tabular}

Table 1. Statistical analysis of the linear and angular differences of the maxillary segments, mandibular body segments and bilateral ramus planes. All data showed in mean \pm SD. $*$ The difference was considered statistically significant if $p \leq 0.05$.

CCWR produce during the orthognathic surgery. Meanwhile, the rigid internal fixation technology dramatically improve the postoperative stability of orthognathic surgery ${ }^{13}$. There exist a trade-off for surgeons between the uncertain postoperative stability and ideal aesthetic outcomes. An accurate and objective evaluation system for the postoperative skeletal stability after CCWR produce is needed to minimize the potential risk.

To evaluate the stability after CCWR, traditional methods were often utilised using two-dimensional lateral cephalometric radiographs, which has several disadvantages such as lack of perspective, errors in projection and superimposition, variations in magnification, voids of information, and errors in positioning the cone head ${ }^{14}$.

Three-dimensional (3D) computer-aided surgical simulation system has been evaluated for its clinical feasibility and accuracy ${ }^{15}$. The construction of $3 \mathrm{D}$ virtual head models enables the morphologic changes of the skeletal segments more visible ${ }^{16}$. The superimposition of $3 \mathrm{D}$ head models realize the quantification of segments movements, which could not been performed on $2 \mathrm{D}$ radiographs. The purpose of this study is to establish a novel 3D measuring method to analyse the postoperative skeletal stability after performing the counter-clockwise rotation of the MMC in patients with skeletal class II deformity.

\section{Results}

Twenty-five patients ( 5 males and 20 females, mean age $23.4 \pm 4.7$ years, range 18 to 29 years) with skeletal class II deformity were included in this retrospective study. They all underwent othognathic surgery thru the CCWR procedure in our department. The linear plane differences in the landmarks represent the changes in position and the differences in the angle planes represent the changes in posture. The differences in the angle between the left and right ramus is shown in Table 1.

It can conclude that the maxilla moved backward and upward postoperatively in the analysis of the difference in measurements between $\mathrm{T} 0$ and $\mathrm{T} 1$, while all measurements in the mandible had shown that it advanced 
horizontally ( $p \leq 0.05$ statistically significant difference thru the ANOVA test). During the operation, the change in the mean pitch plane with counterclockwise rotation was $6.48^{\circ}$ on the maxilla and $4.73^{\circ}$ on the mandible.

Postoperative follow-up showed the linear relapse of the maxillary landmarks between T1, T2 and T3 were less than $0.5 \mathrm{~mm}$, (no statistically significant difference $p \leq 0.05$, ANOVA test), which would mean a very stable result. Compared with immediate postoperative maxillary pitch plane posture, there was average $6.64 \%$ relapse at 3 months follow-up (T2-T1/T1-T0) and 23.46\% relapse at 6 months follow-up (T3-T1/T1-T0). There was no significant angle changes in the roll plane and yaw plane between T1, T2, and T3 models, which is therefore acceptable.

Postoperative follow-up showed that the largest positional linear relapse of the mandibular landmarks were less than $2.0 \mathrm{~mm}$. The distance between the B point and mandibular CP' increased from $71.03 \mathrm{~mm}$ to $73.84 \mathrm{~mm}$ postop. Then it further decreased to $73.49 \mathrm{~mm}$ three months after surgery, continually decreased to $71.84 \mathrm{~mm}$ in the subsequent follow-ups. There was a statistically significant pitch plane relapse of the mandible with an average of $1.86^{\circ}$ between $\mathrm{T} 1$ and $\mathrm{T} 2,3.28^{\circ}$ between $\mathrm{T} 1$ and $\mathrm{T} 3$. $\angle \mathrm{YP}-\mathrm{CP}$ ' showed no significant postural angular changes during the 6-months follow-up.

In conclusion, this novel three-dimensional Position-Posture measuring method can be a useful and reliable tool in measuring the linear and angular changes of target segments and in analyzing the three-dimensional recurrence trend after surgery quamtitatively. Based on the results of the Position-Posture measuring method, it can conclude that the MMC had a clockwise relapse tendency after performing the counter-clockwise procedure for skeletal class II patients, most especially on the mandibular-body segment. Meanwhile, there were no significant changes on each segments on the vertical and transverse dimensions.

\section{Discussion}

Orthognathic surgery using the counterclockwise rotation technique, despite its esthetic and functional advantages, was not used to treat maxillofacial deformities during the mid 1990 s because of the uncertain postsurgical stability ${ }^{1-3}$. Most of the previous studies that we encountered have used a two dimensional cephalometric evaluation to measure the postsurgical stability of the orthognathic surgery. All the facial bone structures are projected on a single coronal or sagittal plane in frontal or lateral cephalograms. The tissue superimposition and the changes of the patient's position may lead to errors in the magnification and unreliability of the linear distance between 2-dimensional landmarks when used as a refs ${ }^{14,17}$.

Computer-assisted-surgery, allowing 3D planning and simulation, offers a new option for 3D measurement and analysis. By reconstructing the skull model, the position changes of bony landmarks and posture changes of bone segments become more visible. However, previous three-dimensional CT measurement system only represent the bony landmarks of craniofacial bone on CT images, but fail to analyze the bone relationship in three dimensional space ${ }^{18,19}$. $\mathrm{Xia}^{20}$ et al. demonstrated a three-dimensional cephalometry protocol. However, the positon and posture of the segment was defined by geometric method, which is different from the real anatomic landmarks. At the mean time, the error would be dispersed to three axes perpendicular to each other and could not reflect real changes enough. Therefore, the novel "position-posture" method was developed to actually address this need.

For any geometric object, four points and three planes are sufficient to define its position and posture in three dimension. The same principle also can be applied to skull bone segments. This study determined four clinically significant anatomical landmarks which could represent the shape and position of the maxillary or mandibular segments and three postural planes to describe the posture and orientation. The linear and angular changes of the postural plane were measured to analyze the positional and postural changes observed every follow-up. The results of this retrospective study show that postoperative skeletal stability of the maxilla after counterclockwise rotation during orthognathic surgery were acceptable. It also showed that the postoperative angular changes in the pitch plane is more prominent than the other two postural planes. The maxilla also had a clockwise rotation tendency. The linear relapse based on the landmarks on the maxilla during the follow-up revealed no statistical significance. The largest linear changes of mandible-body segments is $2.00 \mathrm{~mm}$ at B point, and the pitch plane relapse indicating that the mandible-body segments tend to rotate clockwise compared to bilateral ramus segments at 6 months follow-up.

Many factors including articular diseases, age and sex of the patient, a high angle of the mandibular plane, condylar position, neuromuscular adaptation, instability of fixation, and the amount of forward advancement may contribute to the clockwise postoperative relapse $e^{4,21,22}$. CCWR of MMC can cause stretching of the pterygomasseteric sling, which in turn produces tension on the area. These, in combination with the inferior and posterior traction forces originating from the suprahyoid muscles, tends to rotate the MMC clockwise and promote the relapse. To correct these problems, some technical modifications have been proposed, such as the detachment and sectioning of the pterygomasseteric sling ${ }^{3}$, as well as performing inverted L osteotomy. These interventions can preserve the connection of the pterygoid muscles with the proximal mandibular stump. Meanwhile, the use of computer-aided surgical simulation technology could predicting the treatment outcomes in an accurate and effective way, giving reference for surgeons and patients when making a trade-off between the relapse risk and aesthetics outcomes ${ }^{23-25}$.

In this study, twenty-three patients had a series of TMJ health problems. The magnetic resonance imaging confirmed the different degrees of disc displacement and condylar resorption in these patients, which may contribute to the mandibular-body segments relapse. The Position-Posture measuring method established the mandibular coordinate system presuming that the condylion and coracoid process points remain unchanged after surgery and the positional relationship of the ramus bilaterally is stable. In order to minimize the errors, the plane of angle between the right and left ramus was measured to verify its reliability, with the maximum value under $2^{\circ}$ and no statistically difference $(P>0.05)$. This shows the reliability of the coordinate system of the mandibular model. 


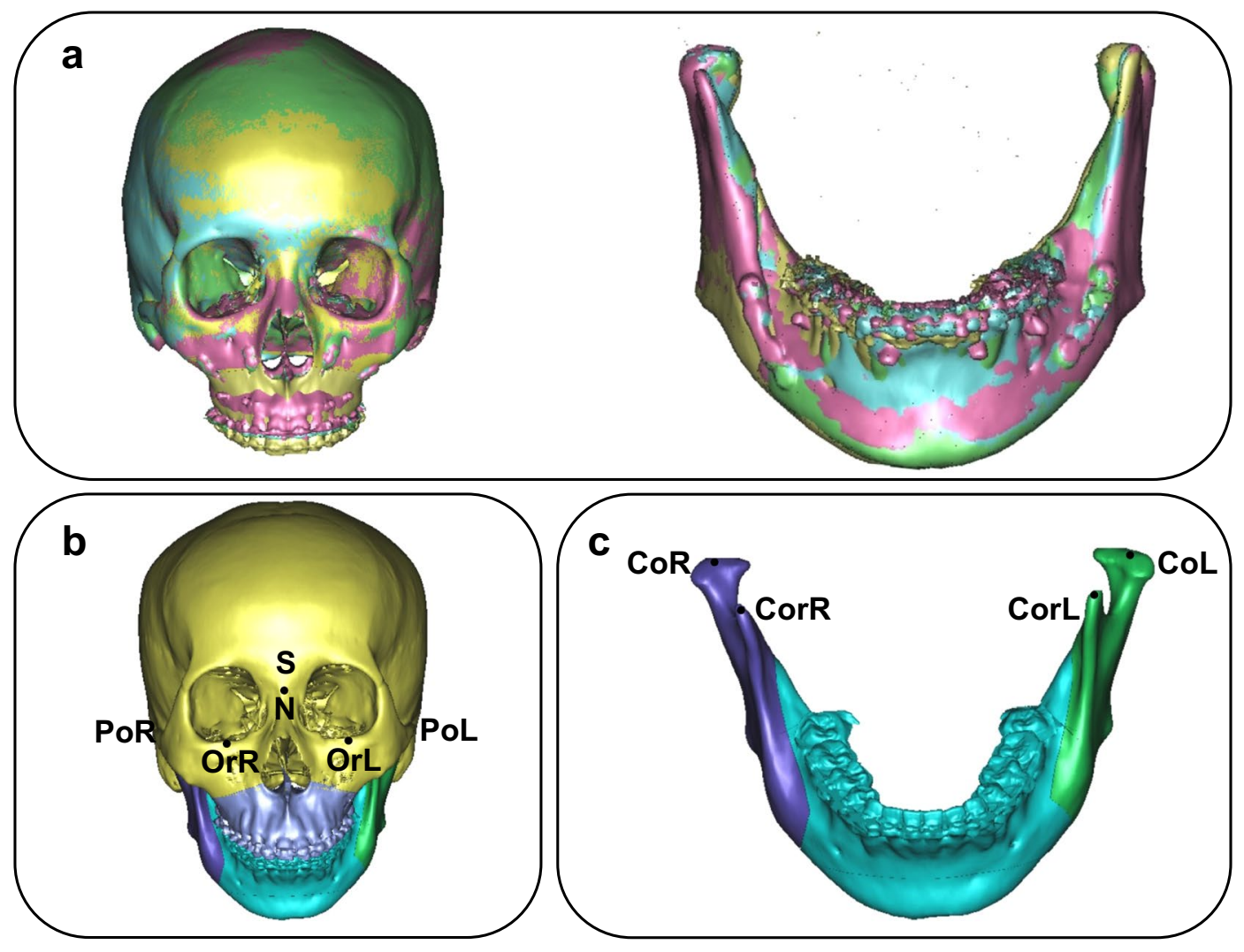

Figure 1. (a) Cranio-maxillofacial and mandibular models of T0-T3 with surface-best-fit method (yellow: T0, pink: T1, blue: T2, green: T3). (b) Bony landmarks of cranio-maxiollofacial were chosen to establish coordinate systems. (c) Bony landmarks of mandibular models were chosen to establish coordinate systems.

The 3D CT scans of patients at different time points were chosen for evaluation because of the excellent visualization of craniofacial skeleton. Although the CT scan examination has higher amounts of radiation than 2D cephalometry and cone beam computed tomography, is generally extremely useful and necessary for pre-surgical simulation and postoperative observation for hard and soft tissue evaluation in clinical application ${ }^{24}$. The 3D CT scan gives more information about important microanatomy structures involving in orthognathic surgery and more detail about soft tissue changes, which could be essential to establish a universal 3D cephalometric system for both bony structures and soft tissue in the future. Meanwhile, this study established a novel measurement method to analyze the postsurgical stability of hard tissues according to the experience of the clinicians. To promote its clinical application, we recommend to select more landmarks to serve as references. The clinical significance will be used to describe the position and posture of the craniofacial hard tissue. We suggest that further research is needed to establish the evaluation systems of bone and soft tissue segments by using the Position-Posture measuring method.

In conclusion, this Position-Posture measuring method has the advantage of revealing the linear and angular changes of the maxillary and mandibular models with great accuracy. It can also help in analyzing the three-dimensional postoperative relapse of hard tissues. By using this method, we can predict the skeletal stability of the maxilla after the counter-clockwise rotation procedure. This can also reveal the tendency for a clockwise relapse of the mandible in the skeletal class II patient.

\section{Methods}

The study was conducted in accordance with the World Medical Association Declaration of Helsinki on medical research ethics. All experimental protocols in this clinical retrospective study were approved by the Ethics Committee of Shanghai Ninth People's Hospital.

Patients. This study was performed in the Department of Oral and Craniomaxillofacial Surgery, Ninth People's Hospital, Shanghai Jiao Tong University School of Medicine, Shanghai, China. From January 2015 to October 2016, 25 patients (5 males and 20 females) with skeletal class II deformity have undergone orthognathic surgery with the CCWR procedure.

The following were the inclusion criteria used to select the candidate patients:

(1) preoperative diagnosis of skeletal class II deformity;

(2) a minimum age of 18 years old;

(3) the use of rigid fixation. 


\begin{tabular}{|c|c|c|}
\hline Abbreviation & Landmark & Anatomical site \\
\hline S & Sella & the geometric center of the sella turcica \\
\hline $\mathrm{N}$ & Nasion & $\begin{array}{l}\text { most posterior point on curvature between frontal bone and nasal } \\
\text { bone in midsagittal plane }\end{array}$ \\
\hline Or L/R & Orbitale & lowest point on infraorbital margin of each orbit \\
\hline Po L/R & Porion & highest midpoint on roof of external auditory meatus \\
\hline A & Subspinale & most posterior point on curve between ANS and prosthion \\
\hline SPC & $\begin{array}{l}\text { Superior central } \\
\text { prosthion }\end{array}$ & $\begin{array}{l}\text { most anterior point in the midline on the alveolar process between } \\
\text { upper central incisors }\end{array}$ \\
\hline SP6L/SP6R & $\begin{array}{l}\text { Superior molar } \\
\text { prosthion }\end{array}$ & $\begin{array}{l}\text { most anterior point in the midline on the alveolar process of the } \\
\text { upper left/right first molar }\end{array}$ \\
\hline B & Supramental & $\begin{array}{l}\text { most posterior point of bony curvature of mandible below } \\
\text { infradentale and above pogonion }\end{array}$ \\
\hline IPC & $\begin{array}{l}\text { Inferior central } \\
\text { prosthion }\end{array}$ & $\begin{array}{l}\text { most anterior point in the midline on the alveolar process between } \\
\text { lower central incisors }\end{array}$ \\
\hline IP6L/IP6R & $\begin{array}{l}\text { Inferior molar } \\
\text { prosthion }\end{array}$ & $\begin{array}{l}\text { most anterior point in the midline on the alveolar process of the } \\
\text { lower left/right first molar }\end{array}$ \\
\hline Co L/R & condylion & most superior point of left/right ondylar head surface \\
\hline Cor L/R & coracoid process & most superior point of left/right coracoid process surface \\
\hline GoL/R & Gonion L/R & $\begin{array}{l}\text { most inferior, posterior, and lateral point on the external angle of } \\
\text { the mandible }\end{array}$ \\
\hline
\end{tabular}

Table 2. Definition of skeletal landmarks.

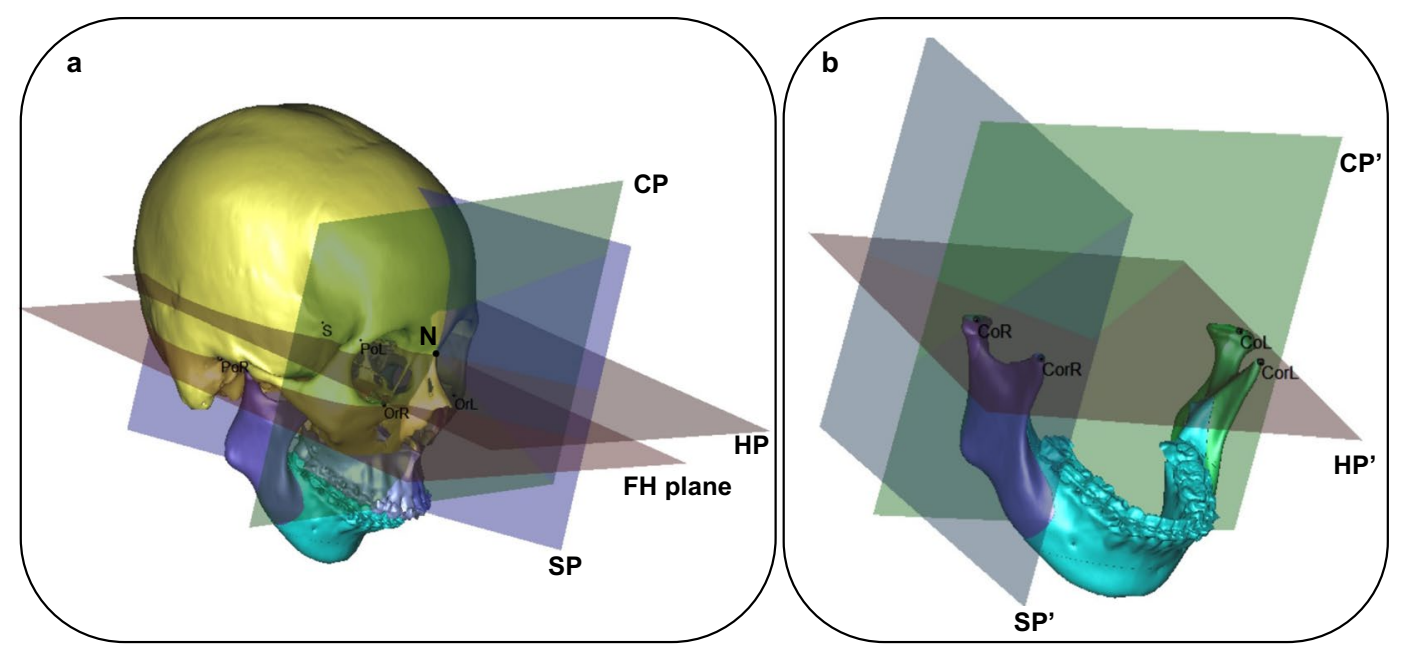

Figure 2. (a) Coordinate systems of cranio-maxiollofacial model with $45^{\circ}$ angle. (b) Coordinate systems of mandibular model with $45^{\circ}$ angle.

Patients were excluded using the following exclusion criteria:

(1) patients diagnosed having a syndrome;

(2) those with either growth or mental retardation.

All patients included in the study accomplished and signed an informed consent.

Pre and post-operative CT (Light speed 32, GE, UK: $1.25 \mathrm{~mm}$ slice thickness) were obtained for all patients 1 week prior to surgery(T0), 3-7days(T1), 3 months(T2), and 6 months(T3) after surgery.

Surgical procedure. Commercially available surgical simulation software SimPlant Pro 11.4 (Materialise Dental, Belgium) was used for the pre-operative planning. All of the personalized virtual surgical planning included counterclockwise rotation of the MMC. All patients underwent orthognathic surgery of the one-piece Lefort I osteotomy and bilateral sagittal split ramus osteotomy (BSSRO) under the guidance of a 3D printed occlusion splints.

Measuring method. The skull model, was separated into two models, the cranio-maxiollofacial model and mandibular model. Through the use of the surface-best fit registration method ${ }^{20}$, the $3 \mathrm{D}$ cranio-maxiollofacial models and mandibular models of T1, T2, and T3 were superimposed on T0 ones (Fig. 1a) consecutively with the aid of the SimPlant Pro software. 


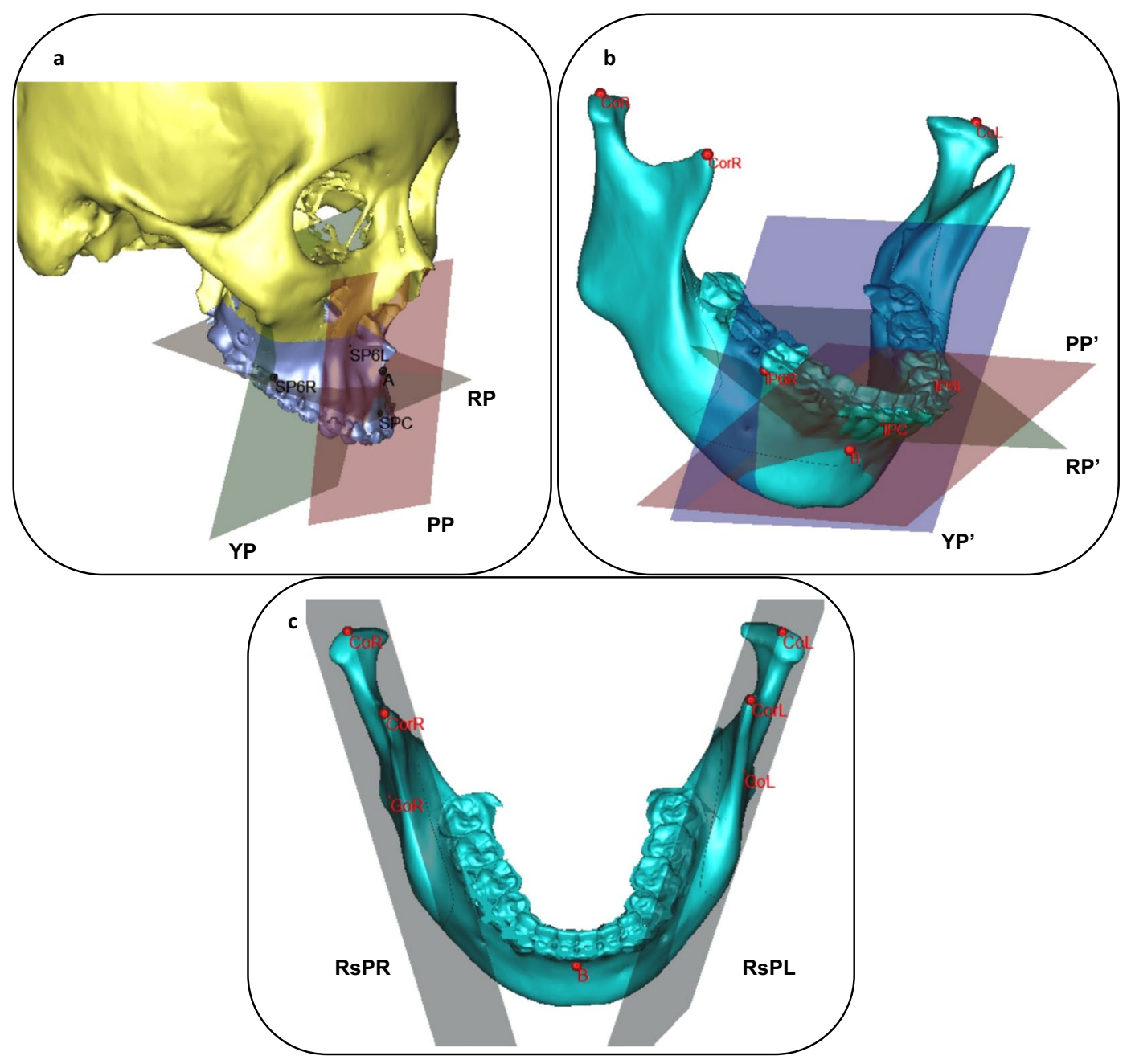

Figure 3. (a) Posture plane (PP, RP and YP) of maxillary segments. (b) Posture plane(PP, RP and YP) of mandible-body segment. (a) Bony landmarks and posture planes of bilateral ramus segments.

\begin{tabular}{|l|l|l|l|l|l|l|l|l|}
\hline value & $\mathbf{A}$ & SPC & SP6L & SP6R & B & IPC & IP6L & IP6R \\
\hline $\mathrm{X}$ & A-SP & SPC-SP & SP6L-SP & SP6R-SP & B-SP' & IPC-SP' & IP6L-SP' & IP6R-SP' \\
\hline Y & A-HP & SPC-HP & SP6L-HP & SP6R-HP & B-HP' & IPC-HP' & IP6L-HP' & IP6R-HP' \\
\hline Z & A-CP & SPC-CP & SP6L-CP & SP6R-CP & B-CP' & IPC-CP' & IP6L-CP' & IP6R-CP' \\
\hline
\end{tabular}

Table 3. Coordinate value of maxillary\&mandibular landmarks in 3-D coordinate system.

Bony landmarks of the cranio-maxiollofacial models (Fig. 1b) and mandibular models (Fig. 1c) were chosen by experienced surgeons to establish the coordinate systems separately (Table 2).

Coordinate system of cranio-maxiollofacial model. Cranial segment, which remain unchanged before and after surgery, was defined as the reference coordinate system of the 3D cranio-maxiollofacial model (Fig. 2a).

The horizontal reference plane (HP): parallel to the FH plane, which was constructed on both sides of Po and left side Or, passing through $\mathrm{N}$.

The midsagittal plane (SP): perpendicular to the horizontal plane passing through $\mathrm{N}$ and $\mathrm{S}$.

The coronal plane (CP): perpendicular to the horizontal and midsagittal plane passing through $\mathrm{N}$.

$\mathrm{N}$ point was defined as the original point of the coordinate system.

Coordinate system of mandibular model. For T1, T2 and T3 mandibular models, Co L/R and Cor L/R remain unchanged after surgery, which could be used to defined the reference coordinate system of mandibular model (Fig. 2b).

The mandible-body horizontal reference plane (HP') was constructed on Co L/R and CorR. 


\begin{tabular}{|l|l|}
\hline Angle & Definition \\
\hline maxillary $\angle \mathrm{PP}-\mathrm{CP}(\mathrm{deg})$ & $\begin{array}{l}\text { the angle between the maxillary CP and maxillary pitch plane, which passing } \\
\text { through 2 points (A, SPC) and normal to SP }\end{array}$ \\
\hline maxillary $\angle \mathrm{RP}-\mathrm{HP}(\mathrm{deg})$ & $\begin{array}{l}\text { the angle between the maxillary HP and maxillary roll plane, which passing } \\
\text { through 2 points (SP6L, SP6R) and normal to CP }\end{array}$ \\
\hline maxillary $\angle \mathrm{YP}-\mathrm{CP}(\mathrm{deg})$ & $\begin{array}{l}\text { the angle between the maxillary CP and maxillary yaw plane, which passing } \\
\text { through 2 points (SP6L, SP6R) and normal to HP }\end{array}$ \\
\hline mandibular $\angle \mathrm{PP}$ '-CP' (deg) & $\begin{array}{l}\text { the angle between the mandibular CP' and mandibular pitch plane, which } \\
\text { passing through 2 points (B, IPC) and normal to SP' }\end{array}$ \\
\hline mandibular $\angle \mathrm{RP}$-HP' (deg) & $\begin{array}{l}\text { the angle between the mandibular HP' and mandibular roll plane, which } \\
\text { passing through 2 points (IP6L, IP6R) and normal to CP' }\end{array}$ \\
\hline mandibular $\angle \mathrm{YP}$ '-CP' (deg) & $\begin{array}{l}\text { the angle between the mandibular CP' and mandibular yaw plane, which } \\
\text { passing through 2 points (IP6L, IP6R) and normal to HP' }\end{array}$ \\
\hline
\end{tabular}

Table 4. Definition of angle between the posture planes and reference planes.

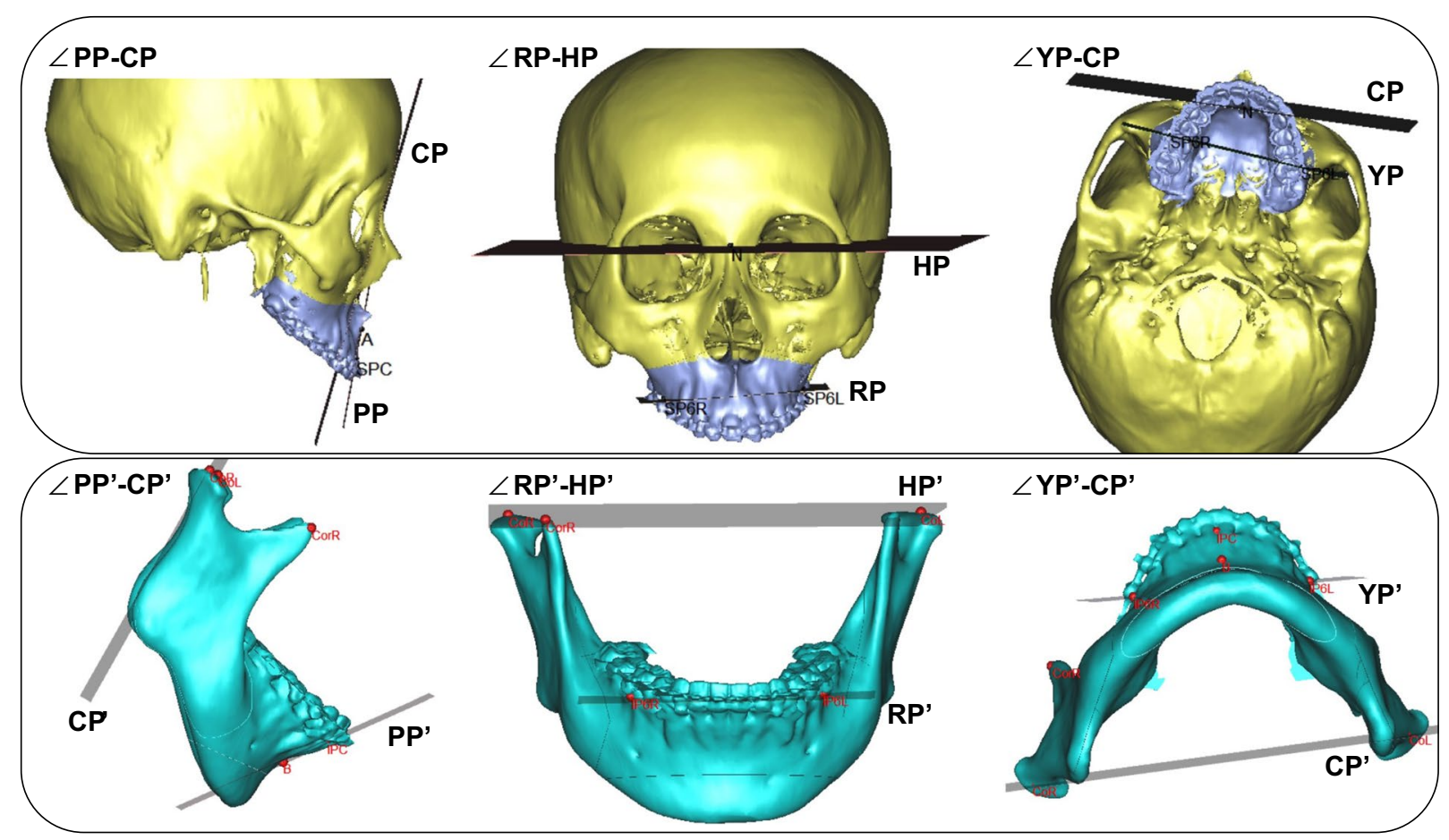

Figure 4. Definition of angle between the posture planes (PP, RP and YP) and reference planes on craniomaxiollofacial model and mandibular model.

The mandible-body midsagittal plane (SP') was drawn perpendicular to the horizontal plane passing through CoR and CorR.

The mandible-body coronal plane (CP') was drawn perpendicular to the horizontal and midsagittal plane passing through CoR.

CoR was defined as the original point of the coordinate system.

In order to precisely measure and analyze the changes, the target cranio-maxiollofacial model was defined as cranial segment and maxillary segment, and the target mandibular model was referred as mandibular-body segment and bilateral ramus segments. We can choose a series of remarkable anatomical landmarks for describing the position of each segments, while three mutually perpendicular planes for describing their posture were also used. Therefore, position and posture of each segment could be defined separately as follows:

Cranial segment: position/posture: Cranial segment, which remain unchanged before and after surgery, was defined as the reference coordinate system of the 3D cranio-maxiollofacial model. So the landmarks of cranio-maxiollofacial model (Fig. 1b) was defined as the position of cranial segment, and the three coordinate planes (Fig. 2a) as its posture.

Maxillary segment: position: In order to describe the position of maxilla, several clinically significant anatomical landmarks were chosen by experienced surgeon. Skeletal landmarks A, SPC, SP6L and SP6R (Table 2) were chosen to describe the position of maxillary segment.

Maxillary segment: posture: Based on the these landmarks, we defined 3 posture planes (Fig. 3a).

yaw plane (YP): passing through 2 points (SP6L, SP6R) and normal to HP;

pitch plane (PP): passing through 2 points $(\mathrm{A}, \mathrm{SPC})$ and normal to SP 
roll plan (RP): passing through 2 points (SP6L, SP6R) and normal to CP

Mandible-body segment: position: In order to describe the position of mandibular-body, several clinically significant anatomical landmarks were chosen by experienced surgeon. Skeletal landmarks B, IPC, IP6L and IP6R (Table 1) were chosen to describe the position of mandibular-body segment.

Mandible-body segment: posture: Based on the these landmarks, we defined 3 posture planes (Fig. 3b).

yaw plane (YP'): passing through 2 points (IP6L, IP6R) and normal to HP';

pitch plane (PP'): passing through 2 points (B, IPC) and normal to SP';

roll plan (RP'): passing through 2 points (IP6L, IP6R) and normal to CP'.

Ramus segments: position: Several clinically significant anatomical landmarks were chosen to describe the position of the ramus segments such as CoL/R, CorL/R and GoL/R. (Table 1).

Ramus segment: posture: Because of the shape of ramus segment, we defined its posture as one plane based on these landmarks (Fig. 3c).

Ramus plane (RsP L/R): passing through 3 points (Co, Cor, Go).

The linear differences of anatomical landmarks could represent the positional change of target maxillary segments and mandibular-body segments on each three-dimensional coordinate system (X, Y, Z) (Table 3).

We also measured the angle differences between the posture planes and reference planes of the cranio-maxiollofacial and mandibular model (Table 4, Fig. 4).

Statistical analysis. All measurements were tabulated and sequenced in the different time frame. Differences between groups were considered significant at $\mathrm{p} \leq 0.05$. One-way ANOVA was used for statistical analysis. All calculations were carried out using the software package SPSS 19.0 (IBM, American).

\section{Data Availability}

The data that supports the findings reported herein are available upon request from the corresponding author.

\section{References}

1. Proffit, W. R., Turvey, T. A. \& Phillips, C. The hierarchy of stability and predictability in orthognathic surgery with rigid fixation: an update and extension. Head \& face medicine 3, 21, https://doi.org/10.1186/1746-160x-3-21 (2007).

2. Wolford, L. M., Chemello, P. D. \& Hilliard, F. Occlusal plane alteration in orthognathic surgery-Part I: Effects on function and esthetics. Am. J. Orthod. Dentofacial Orthop. 106, 304-316, https://doi.org/10.1016/s0889-5406(94)70051-6 (1994).

3. Chemello, P. D., Wolford, L. M. \& Buschang, P. H. Occlusal plane alteration in orthognathic surgery-Part II: Long-term stability of results. Am. J. Orthod. Dentofacial Orthop. 106, 434-440, https://doi.org/10.1016/s0889-5406(94)70066-4 (1994).

4. Reyneke, J. P., Bryant, R. S., Suuronen, R. \& Becker, P. J. Postoperative skeletal stability following clockwise and counter-clockwise rotation of the maxillomandibular complex compared to conventional orthognathic treatment. Br. J. Oral Maxillofac. Surg. 45, 56-64, https://doi.org/10.1016/j.bjoms.2005.12.015 (2007).

5. Gomes, L. R. et al. Three-dimensional quantitative assessment of surgical stability and condylar displacement changes after counterclockwise maxillomandibular advancement surgery: Effect of simultaneous articular disc repositioning. Am. J. Orthod. Dentofacial Orthop. 154, 221-233, https://doi.org/10.1016/j.ajodo.2017.10.030 (2018).

6. Ueki, K. et al. A hypothesis on the desired postoperative position of the condyle in orthognathic surgery: a review. Oral Surg. Oral Med. Oral Pathol. Oral Radiol. 114, 567-576, https://doi.org/10.1016/j.oooo.2011.12.026 (2012).

7. Xi, T., de Koning, M., Berge, S., Hoppenreijs, T. \& Maal, T. The role of mandibular proximal segment rotations on skeletal relapse and condylar remodelling following bilateral sagittal split advancement osteotomies. J. Craniomaxillofac. Surg. 43, 1716-1722, https:// doi.org/10.1016/j.jcms.2015.07.022 (2015).

8. Xi, T. et al. $3 \mathrm{D}$ analysis of condylar remodelling and skeletal relapse following bilateral sagittal split advancement osteotomies. J. Craniomaxillofac. Surg. 43, 462-468, https://doi.org/10.1016/j.jcms.2015.02.006 (2015).

9. Panula, K., Somppi, M., Finne, K. \& Oikarinen, K. Effects of orthognathic surgery on temporomandibular joint dysfunction. A controlled prospective 4-year follow-up study. Int. J. Oral Maxillofac. Surg. 29, 183-187 (2000).

10. Goncalves, J. R. et al. Temporomandibular joint condylar changes following maxillomandibular advancement and articular disc repositioning. J. Oral Maxillofac. Surg. 71, 1759.e1751-1715, https://doi.org/10.1016/j.joms.2013.06.209 (2013).

11. Carvalho Fde, A., Cevidanes, L. H., da Motta, A. T., Almeida, M. A. \& Phillips, C. Three-dimensional assessment of mandibular advancement 1 year after surgery. Am. J. Orthod. Dentofacial Orthop. 137, S53.e51-12; discussion S53-55, https://doi.org/10.1016/j. ajodo.2010.01.017 (2010).

12. Al-Moraissi, E. A. \& Wolford, L. M. Does Temporomandibular Joint Pathology With or Without Surgical Management Affect the Stability of Counterclockwise Rotation of the Maxillomandibular Complex in Orthognathic Surgery? A Systematic Review and Meta-Analysis. J. Oral Maxillofac. Surg. 75, 805-821, https://doi.org/10.1016/j.joms.2016.10.034 (2017).

13. Silvestri, A., Ciaramelletti, M. \& Natali, G. Comparative stability study of wire osteosynthesis versus rigid fixation in the treatment of Class III dentoskeletal deformities. Am. J. Orthod. Dentofacial Orthop. 105, 477-482, https://doi.org/10.1016/s08895406(94)70008-7 (1994).

14. Periago, D. R. et al. Linear accuracy and reliability of cone beam CT derived 3-dimensional images constructed using an orthodontic volumetric rendering program. Angle Orthod. 78, 387-395, https://doi.org/10.2319/122106-52.1 (2008).

15. Xia, J. J. et al. Accuracy of the computer-aided surgical simulation (CASS) system in the treatment of patients with complex craniomaxillofacial deformity: A pilot study. J. Oral Maxillofac. Surg. 65, 248-254, https://doi.org/10.1016/j.joms.2006.10.005 (2007).

16. Xi, T., Schreurs, R., Heerink, W. J., Berge, S. J. \& Maal, T. J. A novel region-growing based semi-automatic segmentation protocol for three-dimensional condylar reconstruction using cone beam computed tomography (CBCT). PloS one 9, e111126, https://doi. org/10.1371/journal.pone.0111126 (2014).

17. Berco, M. et al. Accuracy and reliability of linear cephalometric measurements from cone-beam computed tomography scans of a dry human skull. Am. J. Orthod. Dentofacial Orthop. 136, 17.e11-19; discussion 17-18, https://doi.org/10.1016/j.ajodo.2008.08.021 (2009).

18. Ono, I. et al. Three-dimensional analysis of craniofacial bones using three-dimensional computer tomography. J. Craniomaxillofac. Surg. 20, 49-60 (1992).

19. Hildebolt, C. F., Vannier, M. W. \& Knapp, R. H. Validation study of skull three-dimensional computerized tomography measurements. Am. J. Phys. Anthropol. 82, 283-294, https://doi.org/10.1002/ajpa.1330820307 (1990).

20. Gateno, J., Xia, J. J. \& Teichgraeber, J. F. New 3-dimensional cephalometric analysis for orthognathic surgery. J. Oral Maxillofac. Surg. 69, 606-622, https://doi.org/10.1016/j.joms.2010.09.010 (2011). 
21. Rosen, H. M. Occlusal plane rotation: aesthetic enhancement in mandibular micrognathia. Plast. Reconstr. Surg. 91, 1231-1240; discussion 1241-1234 (1993).

22. Moen, K., Wisth, P. J., Skaale, S., Boe, O. E. \& Tornes, K. Dental or skeletal relapse after sagittal split osteotomy advancement surgery? Long-term follow-up. J. Oral Maxillofac. Surg. 69, e461-468, https://doi.org/10.1016/j.joms.2011.02.086 (2011)

23. Jaisinghani, S., Adams, N. S., Mann, R. J., Polley, J. W. \& Girotto, J. A. Virtual Surgical Planning in Orthognathic Surgery. Eplasty 17, icl (2017).

24. Xia, J. J. et al. Algorithm for planning a double-jaw orthognathic surgery using a computer-aided surgical simulation (CASS) protocol. Part 2: three-dimensional cephalometry. Int. J. Oral Maxillofac. Surg. 44, 1441-1450, https://doi.org/10.1016/j. ijom.2015.06.007 (2015).

25. Hsu, S. S. et al. Accuracy of a computer-aided surgical simulation protocol for orthognathic surgery: a prospective multicenter study. J. Oral Maxillofac. Surg. 71, 128-142, https://doi.org/10.1016/j.joms.2012.03.027 (2013).

\section{Acknowledgements}

This study was supported by Shanghai Jiaotong University "Medical-Engineering Cross Fund" (Grant No. YG2016QN16) and Shanghai Sailing Program (Grant No. 16YF1406800). The study was approved by the ethics committee of the hospital prior to initiation. All patients provided written informed consents.

\section{Author Contributions}

Conceived and designed the experiments: Z.W., H.G. and S.G.S. Performed the experiments and analyzed the data: Z.W. P.Z. and S.S. Wrote the paper: Z.W. and H.G.

\section{Additional Information}

Competing Interests: The authors declare no competing interests.

Publisher's note: Springer Nature remains neutral with regard to jurisdictional claims in published maps and institutional affiliations.

(c) (i) Open Access This article is licensed under a Creative Commons Attribution 4.0 International License, which permits use, sharing, adaptation, distribution and reproduction in any medium or format, as long as you give appropriate credit to the original author(s) and the source, provide a link to the Creative Commons license, and indicate if changes were made. The images or other third party material in this article are included in the article's Creative Commons license, unless indicated otherwise in a credit line to the material. If material is not included in the article's Creative Commons license and your intended use is not permitted by statutory regulation or exceeds the permitted use, you will need to obtain permission directly from the copyright holder. To view a copy of this license, visit http://creativecommons.org/licenses/by/4.0/.

(C) The Author(s) 2019 